\title{
Supply Versus Demand of Health Care Facilities in a Competitive Context: Case of Tuberculosis Care in Douala-Cameroon.
}

\author{
Andre Nana Yakam ( $\square$ nanayakam@yahoo.com ) \\ University of Douala https://orcid.org/0000-0001-6486-6127
}

\section{Prisca Fosso Koncy}

University of Douala

\section{Njocke Martin}

Universite de Douala Faculte des sciences Economique et Gestion Appliquee

Bowong Samuel

Universite de Douala Faculte des Sciences

\section{Fono Aimé Louis}

Universite de Douala Faculte des Sciences

Noeske Jürgen

PNT

Research article

Keywords: health services, health economic, heath policy, spatial analysis, tuberculosis

Posted Date: October 12th, 2020

DOl: https://doi.org/10.21203/rs.3.rs-51754/v1

License: (9) (i) This work is licensed under a Creative Commons Attribution 4.0 International License. Read Full License 


\section{Abstract}

Background: Identifying the socio-economic factors associated with the supply and demand of health facilities (HF), including their spatial model, can contribute to the decision-making of the creation of the HF. This study aims to contribute to this issue in the competitive context of the tuberculosis diagnosis and treatment centres (DTC) in Douala, Cameroon.

Methods: The "observed" number of patients attending the DTCs was compared to the "expected" number, using a bivariate analysis. Multivariate analysis using logistic regression and the analysis of variance were used to identify the factors associated to attendance of DTCs. Spatial analysis was performed to access the spatial-economic model hiding behind the distribution of the DTCs.

Results: There is a spatial-economic model hiding behind the distribution of DTCs in Douala, which dictate their solicitation. The decentralization policy advocated by the national program against tuberculosis (NTP) is not always followed by the patients: The "expected" and the "observed" number of patients in DTCs are not correlated. Patients seeking treatment in DTCs closer to their residence are mainly women and patients from relative poor households, while attendance of DTC was associated with the quality of service

Conclusion: Attendance HF is dictate by the spatial-economic model. The policy of creating HF for TB is not followed by patients in competitive context. Attendance of HF by TB patients is associated with some socioeconomics factors;

\section{Background}

Tuberculosis (TB) is a contagious, airborne disease. TB is one of the top 10 causes of death worldwide. In 2018 there were an estimated 10 million new (incident) TB cases worldwide, of which 1.451 million died - 1.2 million deaths among HIV-negative people and 251000 deaths from TB among HIV-positive people(WHO, 2019). Tuberculosis is a public health problem in many African countries. Cameroon is listed among the high-burden for TB/HIV, with an estimated 47,000 TB cases, which corresponds to an incident rate of 186 (95\% Cl: 121-266) per 100,000 population (WHO, 2019). Douala, the economic capital of Cameroon, counts for $11 \%$ of total population, but registered almost $20 \%$ of the TB prevalence in Cameroon in 2018 (NTP, 2019). Douala is the first TB epidemiology's region in Cameroon (in term of the absolute number of TB cases). Since colonization, the Centre de Pneumophysiologie (CPP), part of Laquintinie Hospital (LH), the largest hospital in the city of Douala, serves as reference centre for TB diagnosis and care. To facilitate access to TB services and relieve the CPP, the National Program against Tuberculosis (NTP), in agreement with the local health authorities, decentralized TB services in 2002, Nowadays, twenty-five other health facilities (HF) of the city have been designated as Centers for Diagnosis and Treatment centers of Tuberculosis (DTC), including 13 public and 12 confessional HF. DTCs are geographically distributed according to WHO recommendations, foreseeing one DTC for 100,000 to 150,000 inhabitants. Before decentralization, more than $60 \%$ of TB patients in the city 
attended the LH. After decentralization, this influx was reduced to approximately $18 \%$ of total cases of reported TB patients, according to the reports of the NTP (unpublished data).

The management of TB is standardized and free-of-charge in Cameroon. According to the logic of the health system, diagnosed TB patients should be distributed almost equitably, according to their residence, over all twenty-five decentralized DTCs, the CPP remaining the specialized center for complicated cases. However, according to the reports of the NTP, the notification of TB cases in the city of Douala varies greatly from one DTC to another. Allover, almost two thirds (2/3) of patients do not attend their designated DTC for diagnosis or treatment (Nana et al, 2013).

The objectives of this study were to describe the spatial distribution of TB patients and DTC in the city of Douala; analyze the level and quality of supply of health care to the TB patients; and identify the socioeconomic factors associated to the "demand" of DTCs.

\section{Methods}

The study population comprises bacteriologically confirmed pulmonary TB (PTB+) cases, permanently ( $\geq 3$ months) residing in Douala who were notified in any one of the functional DTCs, between May, $1^{\text {st }}$ 2011 and April, 30th 2012. During one year, each PTB+ patient consecutively diagnosed in any one of the DTCs was interviewed, his/her residence and DTC of health care were located using a GPS (Global positioning system) - by going to his home (in Douala, as others African cities, most houses and streets do not have proper addresses) - DTC attended and expected to be attended DTC (i.e. closest to the patient's residence) were established for each patient. Corresponding data were represented on a Georeferenced map using QGIS software version 3.4 (QGIS Development Team, 2019). A secondary observational dataset, characterizing each of the DTCs attended by patients was collected, with the participation of the medical staff.

The distribution of DTCs and TB patients' residences in the city of Douala was decrypted to understand the spatial model of competition that lies behind the implementation of DTCs and the choice made by patients for attending a certain DTC. Patient's preferences with respect to DTCs were analyzed and compared with predictions of the health system in other to measure the matching of supply and demand for health care provided to TB patients in the city of Douala. R, version 3.5 (R core Team, 2018), software was used for statistics analysis.

There are two different approaches for understanding the determinants of consumer behavior: economic and psychological approaches. The economic approach that has long exercised its hegemony was based on the work of Stigler (1961) using a cost-benefit approach. In contrast, other authors, including schools based on the management approach emphasize the psychological approach to understand the consumer behavior through the Person-Object-Situation paradigm (POS) developed by Bloch and Richins (1983), Punj and Stewart (1983) and Belk (1975). This paradigm assumes that to determine sources or causes of a phenomenon, in our case the choice of a DTC by a patient, it is necessary to take into account the 
characteristics of the studied object (here DTC), the characteristics of the individual (here TB patient) and the locational characteristics. In our study, these two approaches were used to better understand the choice of DTCs by patients. For the analysis of the factors that determine the demand for health care, we

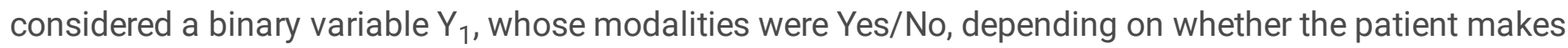
use or not of the DTC closest to his/her home (designated DTC) for treatment. A logistic regression model was used to model the variable $Y_{1}$ as a function of the explanatory variables shown in Table 1. $Y_{1}=0$ if the patient did not use the DTC closest to his home for health care. Else, $Y_{1}=1$. From the supply side, to assess the determinants of attending a DTC by TB patients, the analysis of variance (ANOVA) was used: the discrete quantitative variable $Y_{2}$ representing the number of patients attending a DTC was the dependent variable, and the independent variables are presented in Table 2.

Table 1

Estimation of demand for health care (results of the logistic model)

\begin{tabular}{|c|c|c|}
\hline Variables & Coefficients & z-statistics \\
\hline Sex & $-0.215^{\star}$ & -1.798 \\
\hline Age & $0.0114^{\star \star}$ & 1.974 \\
\hline Head of Household & $-0.582^{\star \star \star}$ & -3.993 \\
\hline Education & $0.323^{\star \star \star}$ & 3.383 \\
\hline Marital status & $0.517 * \star \star$ & 3.817 \\
\hline Family income & $-0.351 * \star \star$ & -4.745 \\
\hline Household size & $-0.0452^{\star \star}$ & -2.142 \\
\hline Water connection & $0.294 * \star$ & -2.39 \\
\hline Constant & 0.438 & -1.112 \\
\hline
\end{tabular}

Table 2

Estimation of supply health care (results from ANOVA)

\begin{tabular}{|c|c|c|c|c|c|}
\hline & Degree of freedom & Sum of squares & mean square & Ficher & P.value \\
\hline HF Standing & 2 & 45155 & 22577 & 4.253 & $0.0360 * \star$ \\
\hline Waiting Time & 2 & 35167 & 17584 & 3.312 & $0.0664^{*}$ \\
\hline Residuals & 14 & 74323 & 5309 & & \\
\hline
\end{tabular}




\section{Results}

During the study period, 2545 PTB + patients permanently residing in Douala were notified: an incidence of approximately $85 / 100000$ people. The residence of 2132 (84\%) among them was mapped, their DTC of care was identified. The $16 \%$ of patients who did not participate in this study did not differ statistically from those who participated in what concerned their distribution by age and sex. Missing cases were distributed proportionally over the entire study area. $61.5 \%$ of diagnosed PTB + patients were men and $38.5 \%$ women, the most affected age group was between 20 and 50 years old (78\%). Figure 1 shows the residences of TB cases by health area (HA). According to the values recorded, the incidence of PTB + cases varies greatly from one HA to another - between $3 / 100,000$ and 482/100, 000. Some HA recorded an incidence rate five times higher than the city average of $85 / 100,000$. The HAs particularly affected by TB were those characterized by high population density (Nkongmondo, Sebenjongo and Bonadiwoto HA) and those that included spontaneous informal settlements of, relatively recent origin (Mabanda, Sodiko, Ndogpassi and Boko HA).

Figure 1 reveals that, at the peripheral areas of the city of Douala - Est and West entrance - DTCs are disposed on a straight line, along $(<0.2 \mathrm{~km})$, the national road number three (3) in respect to the Hotelling model (1929). DTCs located in those area are the less popular (Fig. 2). Further, more than $60 \%$ of TB patients living in peripheral neighborhoods didn't seek for health care in their designated DTCs.

Paradoxically, the population density in these areas is the most important and HA with high TB incidence rate are found there (Nana et al, 2014). In the urban center of the city, the spatial distribution of DTC complies with the provision of circle model of Salop (1979). The number of patients registered in these DTCs (LH, Mbingo, Barcelone, ...) is generally above health system projections (Fig. 2).

Figure 2 shows the attendance of different DTCs by TB patients, comparing the real attendance of each DTC to the expected (the total number of diagnosed TB patients living in the health areas (HA) assumed being covered by the DTC), DTC located in the urban center are more solicited than those in peripheral areas.

According to the result of the logistic regression model (Table 1), women unlike men tend to seek care in health facilities close to their home, particularly those who are heads of their households; this is also the case with TB patients originating from large household size (relatively poor). In contrast, patients living in couple or those with a connection to pipe water (relatively rich) are more likely to seek treatment in DTCs far from their residence. This is also the case with aged TB patients and those with a high level of education.

The analysis of health care delivery for TB patients indicates that the attendance of a health facility (HF) is significantly influenced by the standing of the HF. DTCs located in high standing HF were more popular than others. The size of queue of patients before their treatment was negatively associated with the attendance of DTC (table 2). 


\section{Discussion}

The analysis of the determinants of the demand for health care is important for the formulation of policies and strategies of the health system. Income is undoubtedly one of the most important determinants of demand for health care; particularly in the current context, where TB patients are rather/predominantly recruited from the poorest in society (Nana et al., 2014). Increasing fees can have a negative impact on health services utilization (Lagarde, 2011). Even as there is additional motivation for the personnel of DTC in charge of TB patients by the NTP, in the current context of partial fee exemptions of TB treatment (lab exams paid but medication free), it has been proved that fee exemptions have negative effects on provider motivation and quality of services (Hatt, 2013). Socio-cultural factors, long distances to health facilities and poor quality of care at health facilities have been identified as barriers to utilization of institutional health facilities (Wilunda, 2014).

Besides the direct costs related to medical care, there is another group of determinants of the demand for health care, such as the opportunity costs caused by the disease that may increase the cost of medical treatment. Generally, the health system emphasizes its policy in bringing health facilities closer to the needy population. Several models of spatial competition in the context of location theory take into account the distance between the consumer and goods. Hotelling (1929) and Salop (1979) showed the distance as a factor influencing consumer choice: the consumer will tend to prefer the product that is close to him if he believes that he has the same satisfaction like other products. Nevertheless, the distance can also be understood as the accessibility to a good base on the socioeconomic level of the patient. Landcaster (1979) goes further and sees the distance not only as the geographical point but as a set of product-specific features, characteristics such that any consumer can identify himself as close to or not, according to its appreciation and accessibility to this product.

In the context of large cities such as Douala, where several health facilities (HF) "compete", two models of spatial distribution are possible: a linear distribution, where HF are located in a straight line (Hotelling, 1929) or a circular distribution of HF around patients (Salop 1979). Those two models are implemented in the spatial distribution of HF lodging DTC in Douala, maybe as an artefact, not as result of a conscious distribution policy. A linear model is observed in the peripheral zones, the East and the West entrance of the city and the circular distribution in the urban center. The circular model delivers best results: Patients living in outlying areas of the city preferred seeking treatment in the urban center, although there are HF not far from their homes. The reverse movement is not observed in patients residing in the urban center, where the DTC are distributed circularly.

Patients might be attracted to city center because, considering HFs located there as perfect substitutes, a patient, depending on the idea he/she has of the health facilities, could be embarrassed for the choice of the HF and therefore decide to seek treatment in one or the other, with the possibility to go elsewhere in case of non-availability or long queue. On the other hand, because of the supply induced demand phenomenon, which is a specific feature of health economics, the increase in the supply of health 
infrastructure is driven by an increase in the demand for health care by patients (Richardson J. \& Peacock S.; 2006) which explains the high demand of concentrated health facilities in the urban center.

Whereas, in peripheral areas where a long distance between the HFs is observed, a patient trapped in long queues could not have an alternative solution, especially if it turns out he/she is financially disabled: which characterizes TB patients (Nana et al., 2014). It has been proved that patients attending HF located in the periphery of Douala are those who live or spend most of their time there, such as housewives and housekeepers.

It emerges from our study that TB patients with high level of education, relatively rich, therefore workers, are among those seeking treatment in the city center, whatever their residence. On the other hand, as in many cities, a large number of inhabitants of Douala converge every day to the urban center where they work. If their health can allows them to go about their daily occupation they might avoid the opportunity costs: it might be less expensive to attend a HF in the center - when they are already working there than going back and search in their HA, especially since opening/closing hours play their role.

According to these results, in the absence of regulations on the conditions of reception and referral of patients, the concentration of health facilities in the urban center and industrial zones, with a few lowlevel health facilities in the periphery is the best strategy for the creation of HF in large cities.

From the supply-side, the quality of care provided by health personnel significantly influence the demand. This quality can be perceived by the reception, the availability of medical doctors and nurses or medicines of good quality. Under these conditions, poor quality reception of patients and the nonavailability of conscious and qualified medical staff may discourage patients to return to a health facility. $\mathrm{He} / \mathrm{she}$ might even advise his/her relatives and neighborhood against (Nana et al, 2013). Indeed, the patient, far from being a passive health consumer, is always in the search for best quality of caregivers and health facility to take care of his/her illness.

Good quality of health care in a HF should result in an important number of patients seeking a medical care in this HF, assuming that costs are the same for all HF located in the same geographic area, which is the case for TB health care in DTCs, and that, in case of good appreciation of a HF, the total number of patients attending the DTC of this HF will be equal or greater than the expected one. But the ratio of the number of patients seeking care in the DTC over the expected number (considered here as a measure of supply) varies significantly.

By focusing on the size of the population living in a geographic area to create a DTC, the health system didn't consider the daily migration of population in urban cities, to get to their workplace. It also conceives the health care offer to TB patients, as a market of pure and perfect competition, a form of market in which all hospitals produce an homogeneous "good" (health), consumers (patients) and the producers of the "good" (DTC) are informed, bear no transaction cost and have no inflation on the price. Then, each patient, aware of the homogeneity of supply would therefore not have to brave the distance, with the resulting price to be cured elsewhere if there is a DTC close to his residence. Then almost all patients who 
are taken care by each DTC should reside its immediate surroundings. Which is an ideal in economic theory (Ari Mwachofi, 2011) and far from the realty. Figure 2 shows a situation almost opposite to this theory in the city of Douala: the supply of health care for TB patients in the city of Douala is not perceived as a perfectly competitive market.

Many markets are characterized by monopolistic competition. The HF possesses market power, the power to set a price above marginal cost, although their economic profits remain zero. In Douala, patients consider different HF' label as imperfect substitutes. Thus, rather than comparing the DTC as such, the patient's preference based on the DTC features he has of the HF which houses the DTC.

\section{Conclusion}

In urban cities, where a large variety of facilities offers health services, competing with each other, in the absence of a policy of referral and non-constraint of acceptance of the patient in relation to the distance to his/her residence, the choice of health care facility (HF) follows the law of the market as well as other economic goods. Household's and health facility's characteristics significantly impact the attendance of a HF. Attitudes of TB patients in the city of Douala, facing the offer of health care delivery, are not in line with the predictions of the National TB program (NTP). Although the treatment of TB is free, there are opportunity costs and many other factors, beyond the geographic distance between the patient's residences and a DTC that influence his choice. These determinants of the demand side are gender, household size and household income (poverty). Characteristics that influence the supply side are mainly the standing of health facility and the quality of services, including the patient waiting time before being received. The strategy for creating a DTC at a given geographic location should take into account not only socio-demographic characteristics, but also the behavior and views of the target populations. This study is a contribution for better understanding the attitudes, knowledge and practices of TB patients in relation to the supply of health care of TB in cities such as Douala and an evaluation of the Cameroonian health system policy against TB.

\section{Abbreviations}

HF: Health facilities

DTC: Tuberculosis diagnosis and treatment centres

NTP: National program against tuberculosis

TB: Tuberculosis

WHO: World Health Organization

CPP: Centre de Pneumophysiologie

LH: Laquintinie Hospital 
GPS: Global positioning system

PTB+: Smear positive tuberculosis patient

HA: Health area

\section{Declarations}

\section{Ethics approval and consent to publish:}

Ethics approval for the study and administrative authorisation were granted by the Cameroon national Committee of Ethics (NCE), the National Tuberculosis Programme (NTP) and the Regional Delegation of Public Health. Written informed consent was obtained from all participants.

\section{Consent to publish:}

Not applicable.

\section{Availability of data and materials:}

The datasets used and/or analysed during the current study are available from the corresponding author on reasonable request.

\section{Competing interests:}

The authors declare not to have any competing interests.

\section{Funding:}

Not applicable

\section{Authors' contributions:}

NYA co-drafted protocol, analysed data, co-drafted manuscript

KFP co-drafted protocol, analysed data, co-drafted manuscript

NM co-drafted protocol, co-drafted manuscript

BS co-drafted protocol, co-drafted manuscript 
FLA co-drafted protocol, co-drafted manuscript

NJ co-drafted protocol, co-drafted manuscript

All Authors read and approved the manuscript.

\section{Acknowledgement:}

The authors are very grateful to the German International Cooperation (GIZ), which financed the data collection. The authors thank the health authorities of the Regional Delegation of Public Health in the Littoral Region and the National Tuberculosis Programme for making data available.

\section{References}

1. Ari M, Assaf F (2011), "Health Care Market Deviations from the Ideal Market”, Med SQU. J, August 2011, Vol. 11, Iss. 3, pp. 328-337.

2. Belk Russel W. Situational variables and consumer behavior. J Consum Res. 1975;1(975):2, pp. 15764.

3. Bloch Peter H, Richins ML. A theoretical model for the study of product importance perceptions. Journal of Marketing. 1983;47:69-81.

4. Hatt LE, Makinen M, Madhavan S, Conlon CM. Effects of user fee exemptions on the provision and use of maternal health services: a review of literature. Journal of Health Population Nutrition. 2013;31(4 Suppl 2):67-80.

5. Hotelling H. Stability in competition. Econ J. 1929;39:41-57.

6. Lagarde M, Palmer N. (2011), "The impact of user fees on access to health services in low- and middle-income countries", Cochrane Database of Systematic Reviews, Issue 4, Art. No.:CD009094. DOI: 10.1002/14651858.CD009094.

7. Lancaster KJ. “Consumer Demand: A New Approach”. New - York: Columbia University Press; 1979.

8. Nana YA, Noeske J, Angumua C, Bowong S, Fono LA. Prise en charge des patients souffrant de la tuberculose en milieu urbain: offre de soins et parcours thérapeutique. Santé publique. 2013;25:64753.

9. Nana YA, Noeske J, Dambach P, Bowong S, Fono LA. Spatial analysis of tuberculosis in Douala, Cameroon: Clustering and links with socio-economic status. International journal of tuberculosis lung diseases. 2014;18(3):292-7.

10. Programme national de lute contre la tuberculose (NTP)

Programme national de lute contre la tuberculose (NTP). Website.

HTTP://www.NTP.cm/index.php/component/k2/item/630-situation-epidemiologique-de-latuberculose-au-cameroun. Accessed June 2019. 
11. Punj Girish N, David WS. An interaction framework of consumer decision making. J Consum Res. 1983;10:181-96.

12. QGIS Development Team. (2019), "QGIS Geographic Information System. Open Source Geospatial Foundation Project", http://qgis.osgeo.org.

13. R Core Team. (2018), "R: A language and environment for statistical computing”, R Foundation for Statistical Computing, Vienna, Austria. URL https://www.R-project.org/.

14. Richardson J, Peacock S. Supplier-induced demand: Reconsidering the theories and new Australian evidence. Applied Health Economics Health Policy. 2006;5(2):87-98.

15. Salop (1979), "Monolistic competition with outride goods", Bell Journal of Economics, 10, p. 141156.

16. Stigler George J. The economics of information. Journal of Political Economics. 1961;19:213-25.

17. Wilunda C, Quaglio G, Putoto G, Lochoro P, Dall'Oglio G, Manenti F, et al. A qualitative study on barriers to utilization of institutional delivery services in Moroto and Napak districts, Uganda: implications for programming. BMC Pregnancy Childbirth. 2014;14:259.

18. World Health Organization. (2019), “Global Tuberculosis Report 2019”, ISBN 978-92-4-156571-4 https://apps.who.int/iris/bitstream/handle/10665/329368/9789241565714-eng.pdf, Accessed July 2020.

\section{Figures}

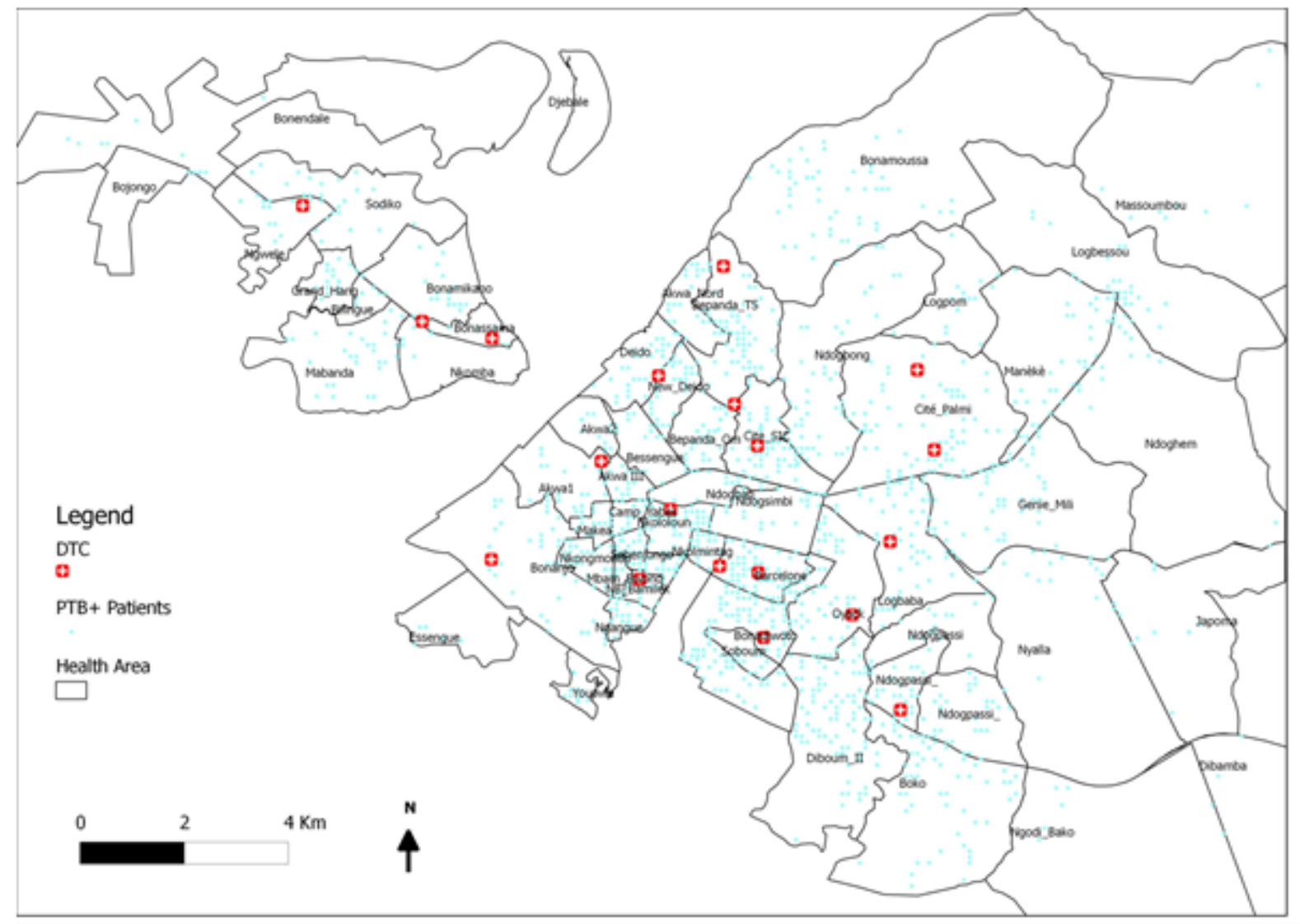


Figure 1

Spatial distribution of smear positive tuberculosis patients and DTCs in Douala, Cameroon.

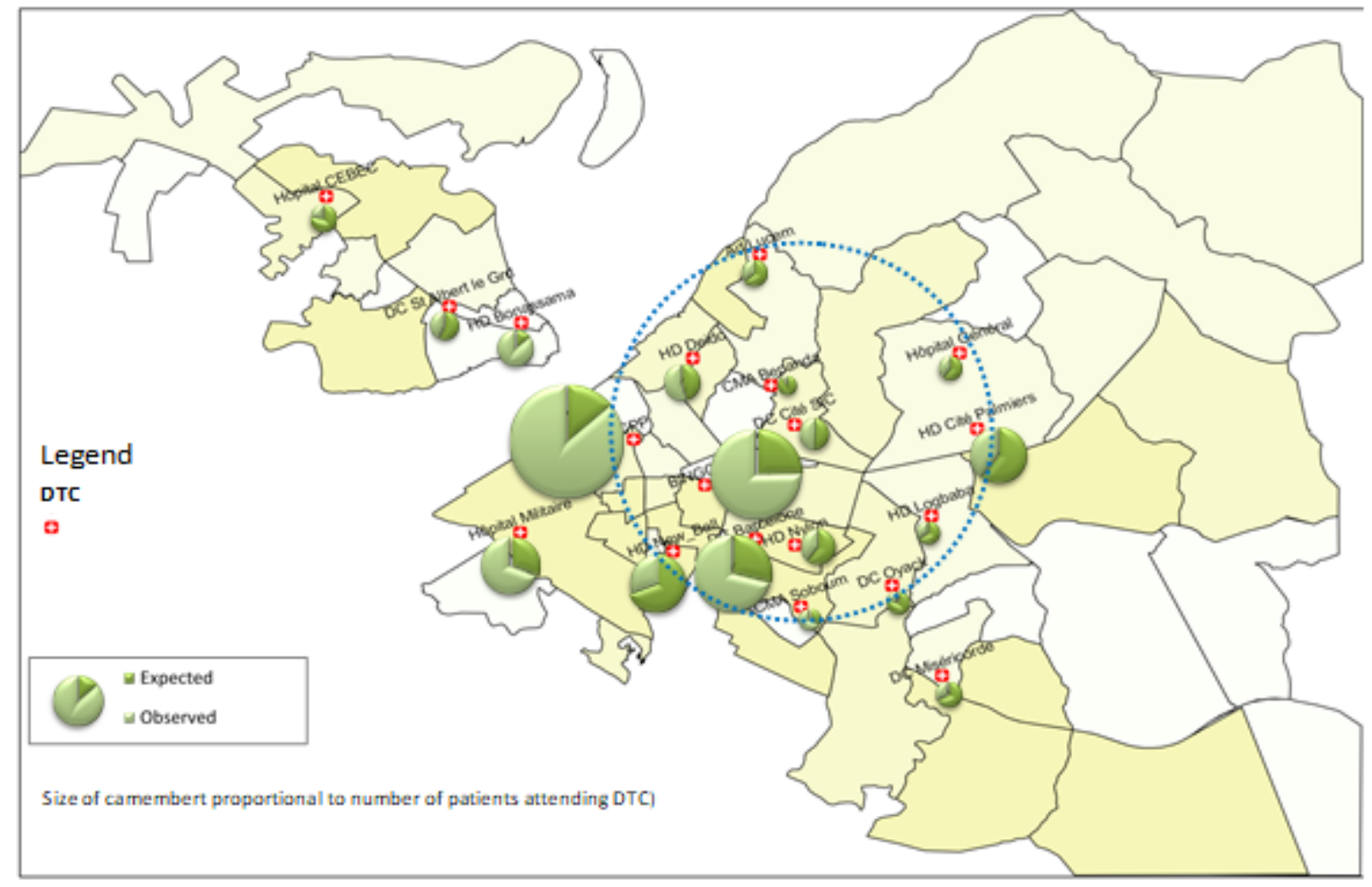

\section{Figure 2}

Spatial model and attendance of DTCs by smear positive tuberculosis patients in Douala, Cameroon. 